TRI-PP-97-5

$\mathrm{MKPH}-\mathrm{T}-97-7$

hep-ph/9702394

\title{
Muon capture by a proton in heavy baryon chiral perturbation theory
}

\author{
Harold W. Fearing and Randy Lewis \\ TRIUMF, 4004 Wesbrook Mall, Vancouver, British Columbia, Canada V6T $2 A 3$ \\ Nader Mobed \\ Department of Physics, University of Regina, Regina, Saskatchewan, Canada S4S 0A2 \\ Stefan Scherer \\ Institut für Kernphysik, Johannes Gutenberg-Universität, J. J. Becher-Weg 45, D-55099 Mainz, \\ Germany
}

(February 21, 1997)

\begin{abstract}
The matrix element for muon capture by a proton is calculated to $\mathcal{O}\left(p^{3}\right)$ within heavy baryon chiral perturbation theory using the new $\mathcal{O}\left(p^{3}\right)$ Lagrangian of Ecker and Mojžiš. External nucleon fields are renormalized using the appropriate definition of the wave function renormalization factor $Z_{N}$. Our expression for $Z_{N}$ differs somewhat from that found in existing literature, but is the one which is consistent with the Lagrangian we use and the one which ensures, within our approach, the nonrenormalization of the vector coupling as required by the conserved vector current. Expressions for the standard muon capture form factors are derived and compared to experimental data and we determine three of the coefficients of the Ecker - Mojžiš Lagrangian, namely, $b_{7}, b_{19}$, and $b_{23}$.
\end{abstract}


Chiral perturbation theory (CHPT) is an effective theory for QCD that allows systematic calculations to be performed whenever external momenta are small with respect to the chiral symmetry breaking scale, $\Lambda_{\chi} \sim 1 \mathrm{GeV}$. The theory was originally formulated for light mesons only [1], but heavy baryons can also be included without sacrificing the small-momentum expansion [2]. As for any effective theory, the Lagrangian of CHPT contains parameters which are not determined by CHPT itself, but which can be inferred from experimental data. Recent reviews of the vast amount of work that has been done with CHPT can be found in Refs. [3 6 ].

The complete Lagrangian for a single nucleon coupling to pions and external fields up to third order in small momenta (denoted $\mathcal{L}_{\pi N}^{E c k M}$ ) has only recently been constructed by Ecker and Mojžiš [7], although calculations for specific processes had been performed earlier. In the present work, we study muon capture by a proton with the new Lagrangian, $\mathcal{L}_{\pi N}^{E c k M}$. The form factors that appear in the muon capture amplitude have been considered previously within heavy baryon CHPT [4, [8], but not with the new Lagrangian $\mathcal{L}_{\pi N}^{E c k M}$.

Our calculation gives explicit expressions for each of the muon capture form factors, in terms of parameters that appear in $\mathcal{L}_{\pi N}^{E c k M}$. We use experimental data to determine the numerical values of the parameters, which are directly transferable to future calculations of other processes where $\mathcal{L}_{\pi N}^{E c k M}$ is used. In particular, the parameters of the present work are a subset of the ones that appear in radiative muon capture by a proton, for which interesting results have been obtained in a recent TRIUMF experiment [9]. A study of radiative muon capture in heavy baryon CHPT is in progress [10].

The external nucleon fields in our calculation are renormalized by defining a wave function renormalization factor, $Z_{N}$, which is the residue of the full nucleon propagator at the pole. The derivation of $Z_{N}$ will be discussed in some detail, since our result differs from that of other groups because of the different Lagrangian. We will show, however, that within our formalism our form of $Z_{N}$ is the one that is necessary to ensure that the vector coupling is not renormalized, a result required by CVC (Conserved Vector Current) theory, which of course ultimately follows from QCD.

The muon capture reaction refers to a muon and proton with negligible relative momentum, interacting to produce a neutron and neutrino,

$$
\mu+p \rightarrow \nu+n .
$$

The 4-momentum transfer in this process, $q=p_{n}-p_{p}$, satisfies

$$
q^{2}=q_{*}^{2} \equiv \frac{-m_{\mu}\left(m_{p}^{2}-m_{n}^{2}+m_{\mu} m_{p}\right)}{m_{p}+m_{\mu}}<0 .
$$

For nonradiative muon capture, $q_{*}^{2}=-0.88 m_{\mu}^{2}$ and so is a small parameter in the context of the CHPT expansion.

The general amplitude for muon capture can be parameterized as follows,

$$
\begin{aligned}
\mathcal{M}= & \frac{-i G_{\beta}}{\sqrt{2}} \bar{u}\left(\mathbf{p}_{\nu}\right) \gamma_{\alpha}\left(1-\gamma_{5}\right) u\left(\mathbf{p}_{\mu}\right) \times \\
& \bar{u}\left(\mathbf{p}_{n}\right)\left[G_{V}\left(q^{2}\right) \gamma^{\alpha}+\frac{i G_{M}\left(q^{2}\right)}{2 m_{N}} \sigma^{\alpha \beta} q_{\beta}-G_{A}\left(q^{2}\right) \gamma^{\alpha} \gamma_{5}-\frac{G_{P}\left(q^{2}\right)}{m_{\mu}} q^{\alpha} \gamma_{5}\right] u\left(\mathbf{p}_{p}\right),
\end{aligned}
$$


where $G_{V}\left(q^{2}\right), G_{M}\left(q^{2}\right), G_{A}\left(q^{2}\right)$ and $G_{P}\left(q^{2}\right)$ are the form factors to be studied, and

$$
\frac{G_{\beta}}{\sqrt{2}}=\frac{G_{F} \cos \theta}{\sqrt{2}}=\frac{g_{W}^{2} \cos \theta}{8 m_{W}^{2}}=(0.8030 \pm 0.0008) \times 10^{-5} \mathrm{GeV}^{-2} \text {. (Ref. [11]) }
$$

Here $m_{N}$ denotes the physical nucleon mass, $G_{F}$ is the Fermi constant, $\theta$ is the Cabibbo angle, and $m_{W}, g_{W}$ are the mass and weak coupling constant of the $\mathrm{W}$ boson. (The protonneutron mass difference is small and will be neglected.) The so-called "second class currents" have not been shown, and do not arise in our CHPT calculation.

With the sign conventions of Eq. (3),$G_{A}\left(q_{*}^{2}\right)$ and $G_{P}\left(q_{*}^{2}\right)$ are positive, matching the conventional positive sign for the parameter " $g_{A}$ " in heavy baryon CHPT. The opposite signs for $G_{A}\left(q^{2}\right)$ and $G_{P}\left(q^{2}\right)$ have almost always been used in non-CHPT studies of radiative and nonradiative muon capture, (e.g. Ref. [12]) and are used in Ref. [11].

Following closely the notation of Ref. [7], the heavy baryon chiral Lagrangian is written in the form,

$$
\mathcal{L}_{\pi N}^{E c k M}=\widehat{\mathcal{L}}_{\pi N}^{(1)}+\widehat{\mathcal{L}}_{\pi N}^{(2)}+\widehat{\mathcal{L}}_{\pi N}^{(3)}
$$

where the superscripts on the right-hand side denote powers in the momentum expansion. The explicit forms of these three terms are given in Eqs. (13), (20) and (23) of Ref. [7]. $\widehat{\mathcal{L}}_{\pi N}^{(1)}$ contains two parameters, $F_{0}$ and $g_{A}$, which correspond to the chiral limits of the pion decay and axial-vector coupling constants, respectively. $\widehat{\mathcal{L}}_{\pi N}^{(2)}$ introduces seven new parameters, labeled $a_{1}, a_{2} \ldots, a_{7}$, two of which will appear in our calculation, plus the nucleon mass in the chiral limit. Another 24 parameters, $b_{1}, b_{2}, \ldots, b_{24}$, arise from $\widehat{\mathcal{L}}_{\pi N}^{(3)}$, and four of these will be present in our study.

The pion field of Ref. [7] will be expressed here in an exponential representation,

$$
U=u^{2}=\exp \left[\frac{i \sigma^{a} \pi^{a}}{F_{0}}\right] \quad, \quad \operatorname{Tr}\left[\sigma^{a} \sigma^{b}\right]=2 \delta^{a b} .
$$

To match our $\mathcal{O}\left(p^{3}\right)$ calculation with the heavy baryon Lagrangian, we will require terms through $\mathcal{O}\left(p^{4}\right)$ from the pure-meson chiral Lagrangian, and for these we use the conventions of Gasser, Sainio and Švarc [13], in particular the terms involving $l_{3}, l_{4}$ given in their Eq. (5.9). In both the meson and baryon sectors of the Lagrangian, the charged weak gauge bosons are included as external fields in the following manner,

$$
\ell_{\mu}=\frac{-g_{W} \cos \theta}{\sqrt{2}}\left(\begin{array}{cc}
0 & W_{\mu}^{+} \\
W_{\mu}^{-} & 0
\end{array}\right) \quad, \quad r_{\mu}=0 .
$$

Throughout our calculation, we write the proton and neutron 4-momenta as

$$
p_{p}^{\alpha}=m_{0 N} v^{\alpha}+k_{p}^{\alpha} \quad, \quad p_{n}^{\alpha}=m_{0 N} v^{\alpha}+k_{p}^{\alpha}+q^{\alpha},
$$

where $m_{0 N}$ is the bare nucleon mass, i.e. the nucleon mass in the chiral limit, and $\alpha$ is the Lorentz index. Any choices for $v^{\alpha}$ and $k_{p}^{\alpha}$ which satisfy the on-shell conditions for the nucleons are valid, and the verification of this reparameterization invariance [14 offers a check on our results. 
In heavy baryon CHPT, the muon capture amplitude takes the form

$$
\mathcal{M}=\bar{u}\left(\mathbf{p}_{\nu}\right) \frac{-i g_{W}}{2 \sqrt{2}} \gamma_{\alpha}\left(1-\gamma_{5}\right) u\left(\mathbf{p}_{\mu}\right) \frac{i}{m_{W}^{2}} \bar{n}_{v}\left(\mathbf{p}_{n}\right) X^{\alpha}(q) n_{v}\left(\mathbf{p}_{p}\right) .
$$

We choose to denote the general field operator appearing in the relativistic Lagrangian by $\psi(x)$ and for the matrix element use the Dirac 4-spinors $u(\mathbf{p})$, with $\mathbf{p}$ being the 3-momentum, which arise in the expansion of the free field. Similarly, $N_{v}(x)$ is the heavy baryon field and $n_{v}(\mathbf{p})$ are the heavy baryon 4 -spinors. $N_{v}(x)$ is obtained by making the usual heavy baryon transformation on the Dirac field operator, i.e.,

$$
N_{v}(x)=\exp \left[i m_{0 N} v \cdot x\right] \frac{1}{2}(1+\psi) \psi(x) .
$$

Some care must be exercised with the normalization. In matrix elements we always use normalized spinors, $\bar{u} u=\bar{n}_{v} n_{v}=1$.

The function $X^{\alpha}(q)$ in Eq. (91) has a one-particle-irreducible term and a pion pole term,

$$
X^{\alpha}(q)=\Gamma_{p W n}^{(r) \alpha}(q)+\Gamma_{p \pi n}^{(r)}(q)\left[\frac{i}{q^{2}-m_{\pi}^{2}}\right] \Gamma_{W \pi}^{(r) \alpha}(q),
$$

where $m_{\pi}$ represents the physical pion mass, and the superscript " $(r)$ " on each of the components means it has been renormalized individually and is finite. To the order considered the renormalized pion propagator has the form of the free propagator, but with the physical mass.

We will now calculate the renormalized components of $X^{\alpha}(q)$, beginning with those from the pure-meson Lagrangian. Working to $\mathcal{O}\left(p^{4}\right)$, the charged-pion, one-particle-irreducible, unrenormalized, amputated, two-point, Green function is the sum of tree-level and one-loop contributions, which we compute using dimensional regularization in $d$ dimensions.

$$
\Gamma_{\pi \pi}\left(q^{2}\right)=i\left(q^{2}-m_{0 \pi}^{2}\right)+\frac{i m_{0 \pi}^{2}}{F_{0}^{2}}\left[2 q^{2} l_{4}-2 m_{0 \pi}^{2}\left(l_{3}+l_{4}\right)+\frac{\left(m_{0 \pi}^{2}-4 q^{2}\right)}{6(4 \pi)^{2}}\left(R+\ln \left(\frac{m_{0 \pi}^{2}}{\mu^{2}}\right)\right)\right] .
$$

Here $m_{0 \pi}$ is the bare pion mass and $R$ contains the divergent piece of the loop graphs,

$$
R \equiv \frac{2}{d-4}-1+\gamma-\ln (4 \pi)+\mathcal{O}(4-d) .
$$

$\Gamma_{\pi \pi}\left(q^{2}\right)$ is related to the pion self energy $\Sigma\left(q^{2}\right)$ via $\Gamma_{\pi \pi}\left(q^{2}\right)=i\left(q^{2}-m_{0 \pi}^{2}-\Sigma\left(q^{2}\right)\right)$. Now to obtain the mass and wave function renormalization and the renormalized propagator we follow standard field theory techniques as described for example in Cheng and Li [15]. Thus we write for the full propagator, to the order to which we are working,

$$
-\frac{1}{\Gamma_{\pi \pi}\left(q^{2}\right)}=\frac{i}{q^{2}-m_{0 \pi}^{2}-\Sigma\left(q^{2}\right)}=\frac{i}{q^{2}-m_{0 \pi}^{2}-\Sigma\left(m_{\pi}^{2}\right)-\left(q^{2}-m_{\pi}^{2}\right) \Sigma^{\prime}\left(m_{\pi}^{2}\right)-\tilde{\Sigma}\left(q^{2}\right)} .
$$

To get the last equation we have expanded the self energy about the point $q^{2}=m_{\pi}^{2}$ so that $\Sigma\left(m_{\pi}^{2}\right)$ and $\Sigma^{\prime}\left(m_{\pi}^{2}\right)$ are respectively the value and derivative of $\Sigma\left(q^{2}\right)$ at that point and $\tilde{\Sigma}$ is 
zero in this particular case, but in general is the residual part which goes to zero at $q^{2}=m_{\pi}^{2}$ at least as fast as $\left(q^{2}-m_{\pi}^{2}\right)^{2}$.

The physical pion mass is obtained from the condition that the propagator have a pole at the physical mass, i.e. that $\Gamma_{\pi \pi}\left(m_{\pi}^{2}\right)=0$, giving $m_{\pi}^{2}=m_{0 \pi}^{2}+\Sigma\left(m_{\pi}^{2}\right)$ or

$$
m_{\pi}^{2}=m_{0 \pi}^{2}\left[1+\frac{2 m_{\pi}^{2}}{F^{2}}\left(l_{3}^{r}(\mu)+\frac{1}{4(4 \pi)^{2}} \ln \left(\frac{m_{\pi}^{2}}{\mu^{2}}\right)\right)\right],
$$

where the parameter $l_{3}$ has been renormalized to absorb the divergence using

$$
l_{3}^{r}(\mu)=l_{3}+\frac{R}{4(4 \pi)^{2}},
$$

and where we have anticipated that the difference between $F_{0}$ and the renormalized value $F$ is of higher order and have expressed all quantities in the large brackets of Eq. (15) in terms of physical quantities.

The full propagator can now be written, to the order to which we are working, as

$$
-\frac{1}{\Gamma_{\pi \pi}\left(q^{2}\right)}=\frac{i}{\left(1-\Sigma^{\prime}\left(m_{\pi}^{2}\right)\right)\left(q^{2}-m_{\pi}^{2}-\frac{\tilde{\Sigma}}{1-\Sigma^{\prime}\left(m_{\pi}^{2}\right)}\right)} \equiv \frac{i Z_{\pi}}{q^{2}-m_{\pi}^{2}-Z_{\pi} \tilde{\Sigma}} .
$$

The value of the multiplicative wave function renormalization constant $Z_{\pi}$ is thus the residue of the propagator at the pole (i.e. at the on-shell point) and is given by

$$
Z_{\pi}=\frac{1}{1-\Sigma^{\prime}\left(m_{\pi}^{2}\right)}=\left[\frac{i\left(q^{2}-m_{\pi}^{2}\right)}{\Gamma_{\pi \pi}\left(q^{2}\right)}\right]_{q^{2}=m_{\pi}^{2}}=1-\frac{2 m_{\pi}^{2}}{F^{2}}\left[l_{4}^{r}(\mu)+\frac{2}{3(4 \pi)^{2}} R-\frac{1}{3(4 \pi)^{2}} \ln \left(\frac{m_{\pi}^{2}}{\mu^{2}}\right)\right] .
$$

The parameter $l_{4}$ has been renormalized using

$$
l_{4}^{r}(\mu)=l_{4}-\frac{R}{(4 \pi)^{2}}
$$

which we will justify below. Note that with this choice $Z_{\pi}$ is itself not finite, which is acceptable since, in contrast to $m_{\pi}^{2}, Z_{\pi}$ is not an observable.

If we define a renormalized pion field by

$$
\pi^{(r)}(x)=\frac{\pi(x)}{\sqrt{Z_{\pi}}},
$$

then the two-point function for this renormalized field is finite,

$$
\Gamma_{\pi \pi}^{(r)}\left(q^{2}\right)=Z_{\pi} \Gamma_{\pi \pi}\left(q^{2}\right)=i\left(q^{2}-m_{\pi}^{2}-Z_{\pi} \tilde{\Sigma}\right)
$$

and the propagator for the renormalized field is the negative inverse

$$
-\frac{1}{\Gamma_{\pi \pi}^{(r)}\left(q^{2}\right)}=\frac{i}{q^{2}-m_{\pi}^{2}-Z_{\pi} \tilde{\Sigma}} .
$$


At $\mathcal{O}\left(p^{4}\right) \tilde{\Sigma}=0$, so that we obtain the renormalized propagator which appears in Eq. (11). The interaction between an unrenormalized pion field and a $W$-boson is obtained from tree-level and one-loop diagrams using the meson chiral Lagrangian, and is

$$
\Gamma_{W \pi}^{\alpha}(q)=\frac{F_{0}}{2} q^{\alpha} g_{W} \cos \theta\left[1+\frac{2 m_{0 \pi}^{2}}{F_{0}^{2}} l_{4}-\frac{4 m_{0 \pi}^{2}}{3\left(4 \pi F_{0}\right)^{2}}\left(R+\ln \left(\frac{m_{0 \pi}^{2}}{\mu^{2}}\right)\right)\right] .
$$

The momentum $q$ flows from the $W$-boson to the pion, which is our convention for muon capture. To get the interaction of a renormalized pion, we multiply by $\sqrt{Z_{\pi}}$ to obtain

$$
\Gamma_{W \pi}^{(r) \alpha}(q)=\frac{F}{2} q^{\alpha} g_{W} \cos \theta
$$

where

$$
F=F_{0}\left[1+\frac{m_{\pi}^{2}}{F^{2}}\left(l_{4}^{r}(\mu)-\frac{1}{(4 \pi)^{2}} \ln \left(\frac{m_{\pi}^{2}}{\mu^{2}}\right)\right)\right] .
$$

The renormalization of $l_{4}$ used in Eq. (19) above is required, since $F$ must be finite. Our normalization is such that $F=92.4 \pm 0.3 \mathrm{MeV}$ [11].

Turning now to the nucleon Lagrangian, we use the nucleon two-point function to determine the wave function renormalization factor and the physical nucleon mass in a fashion analogous to that used for the pion. Up to $\mathcal{O}\left(p^{3}\right)$ we find, for a nucleon with four-momentum $p_{p}=m_{0 N} v+k_{p}$,

$$
\Gamma_{N N}\left(p_{p}\right)=i\left[v \cdot p_{p}-m_{0 N}-\Sigma\left(p_{p}\right)\right]=i\left[v \cdot k_{p}-\Sigma\left(p_{p}\right)\right],
$$

where the tiny effects of isospin splitting (proportional to $m_{d}-m_{u}$ ) have been neglected, and

$$
\begin{gathered}
\Sigma\left(p_{p}\right)=-\frac{k_{p}^{2}}{2 m_{0 N}}-\frac{4 a_{3} m_{0 \pi}^{2}}{m_{0 N}}-\frac{3 g_{A}^{2}}{\left(4 \pi F_{0}\right)^{2}}\left[\frac{3}{4} v \cdot k_{p}\left(m_{0 \pi}^{2}-\frac{2}{3}\left(v \cdot k_{p}\right)^{2}\right)\left(R+\ln \left(\frac{m_{0 \pi}^{2}}{\mu^{2}}\right)-\frac{2}{3}\right)\right. \\
\left.+\frac{\left(v \cdot k_{p}\right)^{3}}{6}+\left(m_{0 \pi}^{2}-\left(v \cdot k_{p}\right)^{2}\right)^{3 / 2}\left(\frac{\pi}{2}+\arcsin \left(\frac{v \cdot k_{p}}{m_{0 \pi}}\right)\right)\right]
\end{gathered}
$$

for $(v \cdot k)^{2}<m_{\pi}^{2}$. In this expression the term involving $a_{3}$ is a contact term coming from a piece of $\widehat{\mathcal{L}}_{\pi N}^{(2)}$, the term with $1 /\left(4 \pi F_{0}\right)^{2}$ comes from loop contributions and the $k_{p}^{2}$ term comes from the term in $\widehat{\mathcal{L}}_{\pi N}^{(2)}$ proportional to $-\partial^{2} / 2 m_{0 N}$ which involves no pions. We choose to include this as part of the interaction rather than part of the free Lagrangian and reserve for the free Lagrangian the $i v \cdot \partial$ term from $\widehat{\mathcal{L}}_{\pi N}^{(1)}$.

For the determination of the physical nucleon mass $m_{N}$ and renormalization constant $Z_{N}$ we need the mass-shell condition, $p_{p}^{2}=m_{N}^{2}$, and $v \cdot p_{p}=m_{N}$, where this second condition is motivated by the form of the lowest order propagator, $i\left(v \cdot p_{p}-m_{0 N}\right)^{-1}$. Both conditions taken together are equivalent to $p_{p}=m_{N} v$ which is what is usually stated in the literature.

$\Sigma\left(p_{p}\right)$ is a function of the four momentum $p_{p}$ and thus implicitly of $v$ and $k_{p}$. This dependence can be written in terms of the scalar variables $v \cdot p_{p}-m_{N}$ and $\left(p_{p}-m_{N} v\right)^{2}$. In the vicinity of the pole at $p_{p}=m_{N} v$ these two variables are respectively first and second order in the (small) distance from the pole. 
We now proceed in exactly the same fashion as done for the pion and obtain formulas for the full nucleon propagator analogous to Eqs. (14) and (17), i.e.,

$$
\begin{aligned}
-\frac{1}{\Gamma_{N N}\left(p_{p}\right)} & =\frac{i}{v \cdot p_{p}-m_{0 N}-\Sigma\left(p_{p}\right)}=\frac{i}{v \cdot p_{p}-m_{0 N}-\Sigma\left(m_{N} v\right)-\left(v \cdot p_{p}-m_{N}\right) \Sigma^{\prime}\left(m_{N} v\right)-\tilde{\Sigma}} \\
& =\frac{i}{\left(1-\Sigma^{\prime}\left(m_{N} v\right)\right)\left(v \cdot p_{p}-m_{N}-\frac{\tilde{\Sigma}}{\left(1-\Sigma^{\prime}\left(m_{N} v\right)\right)}\right)}=\frac{i Z_{N}}{v \cdot p_{p}-m_{N}-Z_{N} \tilde{\Sigma}} .
\end{aligned}
$$

In these equations $\Sigma\left(m_{N} v\right)$ and $\Sigma^{\prime}\left(m_{N} v\right)$ are $\Sigma\left(p_{p}\right)$ and its derivative with respect to $\left(v \cdot p_{p}-m_{N}\right)$ evaluated at $p_{p}=m_{N} v$, i.e. at $\left(v \cdot p_{p}-m_{N}\right)=0$, and $\tilde{\Sigma}$ is the residual which goes to zero at the pole at least as fast as $\left(v \cdot p_{p}-m_{N}\right)^{2}$.

The evaluation of $\Sigma\left(m_{N} v\right), \Sigma^{\prime}\left(m_{N} v\right)$, and $\tilde{\Sigma}$ requires an expansion of Eq. (27) but is relatively straightforward. To the order we are working the $a_{3}$ term contributes only to $\Sigma\left(m_{N} v\right)$ whereas the loop piece contributes to all three. Note in particular that $\tilde{\Sigma}$ is not zero in this case. The $k_{p}^{2}$ term requires some discussion however. We can use $p_{p}-m_{N} v=$ $k_{p}-\left(m_{N}-m_{0 N}\right) v$ to write

$$
\frac{k_{p}^{2}}{2 m_{0 N}}=\frac{\left(m_{N}-m_{0 N}\right)^{2}}{2 m_{0 N}}+\frac{\left(m_{N}-m_{0 N}\right)}{m_{0 N}}\left(v \cdot p_{p}-m_{N}\right)+\frac{\left(p_{p}-m_{N} v\right)^{2}}{2 m_{0 N}} .
$$

The first term on the right hand side contributes to $\Sigma\left(m_{N} v\right)$ but is $\mathcal{O}\left(1 / m_{N}^{3}\right)$, since as we shall see $\left(m_{N}-m_{0 N}\right)$ is $\mathcal{O}\left(1 / m_{N}\right)$, and so can be neglected. The second term however is only $\mathcal{O}\left(1 / m_{N}^{2}\right)$ and will contribute to $\Sigma^{\prime}\left(m_{N} v\right)$. Finally the third term contributes only to $\tilde{\Sigma}$.

One can now continue as before. The physical mass, $m_{N}$, is obtained from the requirement that the propagator have a pole at $p_{p}=m_{N} v$, i.e. at $m_{N}=m_{0 N}+\Sigma\left(m_{N} v\right)$, and we find

$$
m_{N}=m_{0 N}\left[1-\frac{4 a_{3} m_{\pi}^{2}}{m_{N}^{2}}-\frac{3 \pi g_{A}^{2} m_{\pi}^{3}}{2 m_{N}(4 \pi F)^{2}}\right] .
$$

The value of the multiplicative renormalization constant $Z_{N}$ is obtained as the residue of the propagator at the pole and is

$Z_{N}=\frac{1}{\left(1-\Sigma^{\prime}\left(m_{N} v\right)\right)}=\left[\frac{i\left(v \cdot p_{p}-m_{N}\right)}{\Gamma_{N N}\left(p_{p}\right)}\right]_{p_{p}=m_{N} v}=1+\frac{4 a_{3} m_{\pi}^{2}}{m_{N}^{2}}-\frac{9 g_{A}^{2} m_{\pi}^{2}}{4(4 \pi F)^{2}}\left(R+\ln \left(\frac{m_{\pi}^{2}}{\mu^{2}}\right)+\frac{2}{3}\right)$.

As in the pion case, we can now define renormalized nucleon fields,

$$
N_{v}^{(r)}(x)=\frac{N_{v}(x)}{\sqrt{Z_{N}}}
$$

and the two-point function for these renormalized fields is finite $\left(Z_{N}\right.$ itself is not finite).

$$
\Gamma_{N N}^{(r)}\left(p_{p}\right)=Z_{N} \Gamma_{N N}\left(p_{p}\right)
$$


The propagator of the renormalized nucleon field is then

$$
-\frac{1}{\Gamma_{N N}^{(r)}\left(p_{p}\right)}=\frac{i}{v \cdot p_{p}-m_{N}-Z_{N} \tilde{\Sigma}}=\frac{i}{v \cdot k_{p}-\left(m_{N}-m_{0 N}\right)-Z_{N} \tilde{\Sigma}} .
$$

For our calculation the propagator is only needed within loop diagrams, and thus only the leading term in the propagator, $i / v \cdot k_{p}$, is needed. However, the full expression, given in Eq. (34), will be required for the nucleons in tree-level diagrams in more complicated processes.

The expression for the renormalization factor of the nucleon commonly found in the literature [4, 16],

$$
Z_{N}^{\text {lit. }} \equiv 1+\left[\frac{\mathrm{d} \Sigma^{\text {lit. }}\left(v \cdot k_{p}\right)}{\mathrm{d} v \cdot k_{p}}\right]_{v \cdot k_{p}=0}=Z_{N}-\frac{4 a_{3} m_{\pi}^{2}}{m_{N}^{2}}+\mathcal{O}\left(\frac{1}{m_{N}^{3}}\right)
$$

differs somewhat from our result. The extra term we obtain originates in the $\left(m_{N}-m_{0 N}\right)$ term from Eq. (29). It involves $a_{3}$ by virtue of Eq. (30).

The two definitions are not equivalent to the order of our calculation, and in our formalism it is $Z_{N}$, not $Z_{N}^{\text {lit. }}$, which represents the full multiplicative renormalization function for the propagator. Therefore $\sqrt{Z_{N}}$ is the factor which exactly accounts for the renormalization of external nucleons in the calculation of a matrix element and allows us to express all matrix elements in terms of fully renormalized and finite, one-particle-irreducible vertex functions.

One way to see this difference explicitly is to compute $G_{V}\left(q^{2}\right)$. Because the vector current is conserved, the calculation must give $G_{V}(0)=1$ to all orders in the $1 / m_{N}$ expansion. We will see that the use of $\sqrt{Z_{N}}$ for external nucleons in our formalism does satisfy this constraint, whereas $\sqrt{Z_{N}^{\text {lit. }}}$ does not.

The origin of this difference can be traced to a difference in the starting Lagrangian. The Lagrangian used in Refs. [4. [16] and in most previous calculations contains a term which is transformed away using the equation of motion in the form proposed by Ecker and Mojžiš [7]. This term generates an $a_{3}$ term in $Z_{N}$ which just cancels the one we find. Of course the physical results must nevertheless be the same, and in fact one finds that this term in the Lagrangian also generates an additional $a_{3}$ term in the matrix element, so that the final result for, say, $G_{V}$ is the same.

Although $Z_{N}$ is in itself not measurable, it does affect measurable quantities. Thus one must always work within a consistent scheme in which the matrix elements, $Z_{N}$, and all other quantities are calculated consistently from the same Lagrangian.

Clearly there is also some additional freedom in the way the finite parts of $Z_{N}$ are handled, even within a consistent scheme. In principle for example one could even within our formalism define a hybrid renormalization scheme in which $Z_{N}^{\text {lit. }}$ accounts for all of the interactions on the external lines except the one involving $a_{3}$. Then in amplitudes one would have to add the one particle reducible diagrams involving the $a_{3}$ interaction on external legs. Alternatively, all insertions on external legs could be calculated explicitly. While not as elegant or easy to do as the standard scheme used here, either of these approaches presumedly would lead to the same final result. Obviously a value of $Z_{N}$ has meaning only when what it includes and the scheme in which it is to be used is precisely defined. 
Two more components of $X^{\alpha}(q)$ in Eq. (11) remain to be determined. The pion-nucleon vertex, renormalized by including the multiplicative factor $Z_{N} \sqrt{Z_{\pi}}$, is

$$
\begin{aligned}
\Gamma_{p \pi n}^{(r)}(q)= & \frac{-\sqrt{2}}{F} g_{A} S \cdot q\left(1+\frac{4 a_{3} m_{\pi}^{2}}{m_{N}^{2}}\right)-\frac{\sqrt{2} g_{A}}{8 m_{N}^{2} F} q \cdot\left(2 k_{p}+q\right) S \cdot\left(2 k_{p}+q\right) \\
& -\frac{4 \sqrt{2}}{(4 \pi F)^{2} F} S \cdot q m_{\pi}^{2}\left[b_{17}^{r}(\mu)-\frac{b_{19}}{2}-\frac{g_{A}^{3}}{4}-\frac{g_{A}}{4}\left(1+2 g_{A}^{2}\right) \ln \left(\frac{m_{\pi}^{2}}{\mu^{2}}\right)\right],
\end{aligned}
$$

and the $W$-nucleon vertex, including the factor $Z_{N}$, is

$$
\begin{aligned}
\Gamma_{p W n}^{(r) \alpha}(q)= & \frac{-i g_{W} \cos \theta}{2 \sqrt{2}} \widetilde{\Gamma}_{p W n}^{(r) \alpha}(q), \\
\widetilde{\Gamma}_{p W n}^{(r) \alpha}(q)= & \left(v^{\alpha}-2 g_{A} S^{\alpha}\right)\left(1+\frac{4 a_{3} m_{\pi}^{2}}{m_{N}^{2}}\right) \\
& +\frac{1}{2 m_{N}}\left[\left(2 k_{p}+q\right)^{\alpha}+2 g_{A} S \cdot\left(2 k_{p}+q\right) v^{\alpha}+8 i a_{6} \epsilon^{\alpha \beta \gamma \delta} q_{\beta} v_{\gamma} S_{\delta}\right] \\
& +\frac{g_{A} q^{2}}{4 m_{N}^{2}} S^{\alpha}+\frac{4}{m_{N}^{2}}\left(a_{6}-\frac{1}{8}\right) i \epsilon^{\alpha \beta \gamma \delta} q_{\beta} k_{p_{\gamma}} S_{\delta}+\frac{g_{A}}{2 m_{N}^{2}}\left[S \cdot\left(k_{p}+q\right) k_{p}^{\alpha}+S \cdot k_{p}\left(k_{p}+q\right)^{\alpha}\right] \\
& -\frac{g_{A}}{4 m_{N}^{2}} i \epsilon^{\alpha \beta \gamma \delta} q_{\beta} v_{\gamma} k_{p_{\delta}}-\frac{2 q^{2} v^{\alpha}}{(4 \pi F)^{2}}\left[b_{7}^{r}(\mu)+\frac{1}{12}\left(1+5 g_{A}^{2}\right) \ln \left(\frac{m_{\pi}^{2}}{\mu^{2}}\right)\right] \\
& -\frac{8 m_{\pi}^{2}}{(4 \pi F)^{2}} S^{\alpha}\left[b_{17}^{r}(\mu)-\frac{g_{A}}{4}\left(1+2 g_{A}^{2}\right) \ln \left(\frac{m_{\pi}^{2}}{\mu^{2}}\right)\right] \\
& -\frac{2 b_{23}}{(4 \pi F)^{2}}\left(S \cdot q q^{\alpha}-q^{2} S^{\alpha}\right)-\frac{q^{2} v^{\alpha}}{18(4 \pi F)^{2}}\left(1+17 g_{A}^{2}\right)+\frac{2 g_{A}^{3} m_{\pi}^{2}}{(4 \pi F)^{2}} S^{\alpha} \\
& +\frac{2 v^{\alpha}}{(4 \pi F)^{2}}\left[\frac{m_{\pi}^{2}}{3}\left(1+2 g_{A}^{2}\right)-\frac{q^{2}}{12}\left(1+5 g_{A}^{2}\right)\right] \int_{0}^{1} \mathrm{~d} x \ln \left(1-x(1-x) \frac{q^{2}}{m_{\pi}^{2}}\right) \\
& -\frac{4 \pi g_{A}^{2} m_{\pi}}{(4 \pi F)^{2}} i \epsilon^{\alpha \beta \gamma \delta} q_{\beta} v_{\gamma} S_{\delta} \int_{0}^{1} \mathrm{~d} x \sqrt{1-x(1-x) \frac{q^{2}}{m_{\pi}^{2}}} \cdot
\end{aligned}
$$

We have used the standard definition,

$$
S^{\alpha}=\frac{i}{2} \gamma_{5} \sigma^{\alpha \beta} v_{\beta}
$$

Notice that we have chosen to write Eqs. (36) and (38) in terms of the physical pion decay constant and the physical masses, rather than $F_{0}$ and the bare masses. The remaining integrals in Eq. (38) can be done analytically for any value of $q^{2}$, taking into account the appropriate boundary conditions in the propagators. For example, at the muon capture point they become

$$
\begin{aligned}
& \int_{0}^{1} \mathrm{~d} x \ln \left(1-x(1-x) \frac{q_{*}^{2}}{m_{\pi}^{2}}\right)=-2+y\left(q_{*}^{2}\right) \ln \left(\frac{y\left(q_{*}^{2}\right)+1}{y\left(q_{*}^{2}\right)-1}\right), \\
& 2 \int_{0}^{1} \mathrm{~d} x \sqrt{1-x(1-x) \frac{q_{*}^{2}}{m_{\pi}^{2}}}=1+\sqrt{\frac{-q_{*}^{2}}{4 m_{\pi}^{2}}} y^{2}\left(q_{*}^{2}\right) \operatorname{arccsc}\left(y\left(q_{*}^{2}\right)\right)
\end{aligned}
$$


with

$$
y\left(q_{*}^{2}\right) \equiv \sqrt{1-\frac{4 m_{\pi}^{2}}{q_{*}^{2}}}
$$

The effects of renormalization on all of the parameters were studied by Ecker [17] using heat kernel techniques. The form appropriate to the Lagrangian we use can be found in Ecker and Mojžišs [7], Table 1 . We have used their results

$$
\begin{gathered}
b_{7}^{r}(\mu)=b_{7}+\frac{1}{12}\left(1+5 g_{A}^{2}\right) R \\
b_{17}^{r}(\mu)=b_{17}-\frac{g_{A}}{4}\left(1+2 g_{A}^{2}\right) R
\end{gathered}
$$

plus the fact that $b_{19}$ and $b_{23}$ do not get renormalized. This ensures that $\Gamma_{p \pi n}^{(r)}(q)$ and $\Gamma_{p W n}^{(r) \alpha}(q)$ are finite and do not depend on the renormalization scale $\mu$.

To obtain expressions for the muon capture form factors, we compare the two forms for the amplitude, given in Eqs. (3) and (9). This comparison requires the well-known momentum dependence of relativistic 4-spinors,

$$
u(\mathbf{p})=\left[\frac{m_{N}+\not p}{\sqrt{2 m_{N}\left(m_{N}+E\right)}}\right] u(\mathbf{0})
$$

where $E \equiv \sqrt{m_{N}^{2}+\mathbf{p}^{2}}$, and also their relation to the normalized heavy baryon 4-spinors,

$$
n_{v}(\mathbf{p})=\sqrt{\frac{2 m_{N}}{m_{N}+v \cdot p_{p}}} \frac{(1+\psi)}{2} u(\mathbf{p})=\left[1-\frac{\not p_{p}}{2 m_{N}}+\frac{\left(m_{N}-m_{0 N}\right)}{2 m_{N}}+\frac{k_{p}^{2}}{8 m_{N}^{2}}+\mathcal{O}\left(\frac{1}{m_{N}^{3}}\right)\right] u(\mathbf{p})
$$

as implied by Eq. (10) and the remark about normalization following it. Eq. (46) is sufficient to rewrite Eq. (9) in the form of Eq. (3). To reverse the procedure and rewrite Eq. (3) in the form of Eq. (9), note that for the specific choice of $v^{\alpha}, v^{0}=1$ and $v^{i}=0$ where $i=1,2,3$, we have $n_{v}(\mathbf{p})=u(\mathbf{0})$. This allows us to write, for that choice of $v^{\alpha}$,

$$
u(\mathbf{p})=\left[\frac{m_{N}+\not p}{\sqrt{2 m_{N}\left(m_{N}+E\right)}}\right] n_{v}(\mathbf{p})=\left[1+\frac{\not p_{p}}{2 m_{N}}-\frac{\left(m_{N}-m_{0 N}\right)}{2 m_{N}}+\frac{k_{p}^{2}}{8 m_{N}^{2}}+\mathcal{O}\left(\frac{1}{m_{N}^{3}}\right)\right] n_{v}(\mathbf{p}) .
$$

The choice of $v^{\alpha}$ has no physical consequences, since the parameter $k_{p}^{\alpha}$ will adapt itself to the chosen $v^{\alpha}$ according to Eq. (8). Also in some cases the algebra becomes simpler with the choice of a particular Lorentz frame, such as the lab frame where the muon and proton are stationary.

Whether or not such simplifying choices are made, the results are the same and we arrive at the following expressions for the muon capture form factors. 


$$
\begin{aligned}
G_{V}\left(q^{2}\right)= & -\left(a_{6}-\frac{1}{8}\right) \frac{q^{2}}{m_{N}^{2}}-\frac{q^{2}}{18(4 \pi F)^{2}}\left(1+17 g_{A}^{2}\right) \\
& -\frac{2 q^{2}}{(4 \pi F)^{2}}\left[b_{7}^{r}(\mu)+\frac{1}{12}\left(1+5 g_{A}^{2}\right) \ln \left(\frac{m_{\pi}^{2}}{\mu^{2}}\right)\right] \\
& +\frac{2}{(4 \pi F)^{2}}\left[\frac{m_{\pi}^{2}}{3}\left(1+2 g_{A}^{2}\right)-\frac{q^{2}}{12}\left(1+5 g_{A}^{2}\right)\right] \int_{0}^{1} \mathrm{~d} x \ln \left(1-x(1-x) \frac{q^{2}}{m_{\pi}^{2}}\right) \\
G_{M}\left(q^{2}\right)= & 4 a_{6}-1-\frac{4 \pi g_{A}^{2} m_{\pi} m_{N}}{(4 \pi F)^{2}} \int_{0}^{1} \mathrm{~d} x \sqrt{1-x(1-x) \frac{q^{2}}{m_{\pi}^{2}}}, \\
G_{A}\left(q^{2}\right)= & g_{A}+\frac{4 a_{3} g_{A} m_{\pi}^{2}}{m_{N}^{2}}-\frac{g_{A}^{3} m_{\pi}^{2}}{(4 \pi F)^{2}} \\
& +\frac{4 m_{\pi}^{2}}{(4 \pi F)^{2}}\left[b_{17}^{r}(\mu)-\frac{g_{A}}{4}\left(1+2 g_{A}^{2}\right) \ln \left(\frac{m_{\pi}^{2}}{\mu^{2}}\right)\right]-\frac{b_{23} q^{2}}{(4 \pi F)^{2}}, \\
G_{P}\left(q^{2}\right)= & \frac{2 m_{\mu} m_{N}}{\left(m_{\pi}^{2}-q^{2}\right)}\left[G_{A}\left(q^{2}\right)-\frac{m_{\pi}^{2}}{(4 \pi F)^{2}}\left(2 b_{19}-b_{23}\right)\right] .
\end{aligned}
$$

To set the stage for our later calculation of radiative muon capture 10 it is necessary to evaluate parameters appearing in these expressions by comparison with other known experimental quantities. We do this in a fashion analogous to that of Ref. 8]. To extract numerical values for the parameters, we need only the first two terms in a $q^{2}$ expansion,

$$
G_{X}\left(q^{2}\right) \equiv G_{X}(0)\left[1+\frac{q^{2}}{6}\left\langle r^{2}\right\rangle_{X}+\mathcal{O}\left(q^{4}\right)\right] .
$$

It is clear from Eq. (48) that $G_{V}(0)=1$, as required by conservation of the vector current. The $a_{3}$ term in the first line of Eq. (38) which arose from $Z_{N}$ just cancels the similar term coming from the $\left(m_{N}-m_{0 N}\right)$ factor of Eqs. (46) or (47) which are used in the extraction of $G_{V}\left(q^{2}\right)$.

Neglecting the electron mass, experimental data from neutron decay 11 give $G_{A}(0)=$ $1.2601 \pm 0.0025$.

The value of $G_{M}(0)$ is related to the nucleon magnetic moments, and implies a numerical value for $a_{6}$.

$$
\kappa_{p}-\kappa_{n}=G_{M}(0)=4 a_{6}-1-\frac{4 \pi m_{\pi} m_{N} G_{A}^{2}(0)}{(4 \pi F)^{2}}
$$

where $\kappa_{p}=1.7928$ and $\kappa_{n}=-1.9130$ are the anomalous magnetic moments [1]. This gives

$$
a_{6}=1.661 \pm 0.004
$$

where the error is dominated by the uncertainty in $F$ and where we have used the average nucleon mass and the charged pion mass in the evaluations. This is in agreement with a recent precision determination of the $\mathcal{L}_{\pi N}^{(2)}$ parameters [18]. It should be noted that the error in Eq. (54) does not contain any uncertainty relating to the convergence of the heavy baryon expansion. For example, if we omit the last term in Eq. (53), corresponding to 
the neglect of $\widehat{\mathcal{L}}_{\pi N}^{(3)}$, then the predicted value of $a_{6}$ is reduced by $30 \%$. The size of this reduction provides some indication of the convergence of the chiral expansion, at least for this particular quantity.

The muon capture form factors of Eqs. (48-51) are related by CVC to the familiar electromagnetic form factors via

$$
\begin{aligned}
G_{V}\left(q^{2}\right) & =F_{1}^{p}\left(q^{2}\right)-F_{1}^{n}\left(q^{2}\right), \\
G_{M}\left(q^{2}\right) & =\kappa_{p} F_{2}^{p}\left(q^{2}\right)-\kappa_{n} F_{2}^{n}\left(q^{2}\right) .
\end{aligned}
$$

Also, for $N=p, n$

$$
\begin{aligned}
& \mathcal{G}_{E}^{N}\left(q^{2}\right) \equiv F_{1}^{N}\left(q^{2}\right)+\frac{q^{2}}{4 m_{N}^{2}} \kappa_{N} F_{2}^{N}\left(q^{2}\right) \\
& \mathcal{G}_{M}^{N}\left(q^{2}\right) \equiv F_{1}^{N}\left(q^{2}\right)+\kappa_{N} F_{2}^{N}\left(q^{2}\right)
\end{aligned}
$$

A simple parameterization which fits the electromagnetic data reasonably well [19] is the dipole,

$$
\mathcal{G}_{E}^{n}\left(q^{2}\right) \approx 0 \quad, \quad \mathcal{G}_{E}^{p}\left(q^{2}\right) \approx \frac{\mathcal{G}_{M}^{p}\left(q^{2}\right)}{1+\kappa_{p}} \approx \frac{\mathcal{G}_{M}^{n}\left(q^{2}\right)}{\kappa_{n}} \approx\left(1-\frac{q^{2}}{0.71 \mathrm{GeV}^{2}}\right)^{-2}
$$

This dipole approximation gives

$$
\begin{aligned}
\left\langle r^{2}\right\rangle_{V} & =0.41 \mathrm{fm}^{2}, \\
\left\langle r^{2}\right\rangle_{M} & =0.72 \mathrm{fm}^{2} .
\end{aligned}
$$

The prediction of our CHPT calculation for $\left\langle r^{2}\right\rangle_{M}$ comes from taking the $q^{2}$ expansion of $G_{M}\left(q^{2}\right)$, as given in Eq. (49). We obtain

$$
\left\langle r^{2}\right\rangle_{M}=\frac{2 \pi m_{N} G_{A}^{2}(0)}{m_{\pi}(4 \pi F)^{2} G_{M}(0)}=0.523 \pm 0.004 \mathrm{fm}^{2} .
$$

The CHPT prediction for $\left\langle r^{2}\right\rangle_{M}$ is about 30\% smaller than the dipole estimate, which is not inconsistent with our expectation for the effect of truncating the chiral expansion at $\mathcal{O}\left(p^{3}\right)$, as discussed above for $a_{6}$ itself. Using the dipole prediction for $\left\langle r^{2}\right\rangle_{V}$ as input to Eq. (48), we can obtain a value for the parameter $b_{7}^{r}$.

$$
\begin{aligned}
\left\langle r^{2}\right\rangle_{V}= & \frac{3}{4 m_{N}^{2}}-\frac{6 a_{6}}{m_{N}^{2}}-\frac{1+7 G_{A}^{2}(0)}{(4 \pi F)^{2}}-\frac{12}{(4 \pi F)^{2}}\left[b_{7}^{r}(\mu)+\frac{1}{12}\left(1+5 G_{A}^{2}(0)\right) \ln \left(\frac{m_{\pi}^{2}}{\mu^{2}}\right)\right] \\
& \Rightarrow b_{7}^{r}\left(m_{N}\right)=-0.53 \pm 0.02 .
\end{aligned}
$$

Errors due to the chiral truncation or to the uncertainty in the dipole prediction for $\left\langle r^{2}\right\rangle_{V}$ are not shown, though in fact they are probably much larger than the other uncertainties included.

The quantity $\left\langle r^{2}\right\rangle_{A}$ has been measured in pion electroproduction [20],

$$
\left\langle r^{2}\right\rangle_{A}=0.35 \pm 0.06 \mathrm{fm}^{2}
$$


and also in antineutrino-nucleon scattering [21],

$$
\left\langle r^{2}\right\rangle_{A}=0.42 \pm 0.04 \mathrm{fm}^{2} .
$$

Some corrections to the electroproduction analysis related to non-zero pion mass actually bring that value into much closer agreement with the neutrino scattering result [22]. With Eq. (50) and the latter value of $\left\langle r^{2}\right\rangle_{A}$, we obtain an estimate of $b_{23}$,

$$
b_{23}=-\frac{1}{6}(4 \pi F)^{2}\left\langle r^{2}\right\rangle_{A} G_{A}(0)=-3.1 \pm 0.3
$$

In principle, $b_{19}$ can now be determined from the experimental value of $\left\langle r^{2}\right\rangle_{P}$, which is, however, not very precise. Instead, we observe that the pion-nucleon vertex function $\Gamma_{p \pi n}^{(r)}(q)$ is related to the renormalized pion nucleon coupling constant via

$$
-\sqrt{2} g_{\pi N N} \bar{u}\left(\mathbf{p}_{n}\right) \gamma_{5} u\left(\mathbf{p}_{p}\right)=\bar{n}_{v}\left(\mathbf{p}_{n}\right) \Gamma_{p \pi n}^{(r)}(q) n_{v}\left(\mathbf{p}_{p}\right)
$$

and proceed in a fashion analogous to Ref. [4]. Applying the same evaluation to this as was used for the other couplings we find

$$
g_{\pi N N}=\frac{m_{N}}{F}\left(G_{A}(0)-\frac{m_{\pi}^{2} b_{19}}{8 \pi^{2} F^{2}}\right) .
$$

Thus we see that $b_{19}$ is related to the so-called Goldberger-Treiman discrepancy

$$
1-\frac{m_{N} G_{A}(0)}{F g_{\pi N N}} \text {. }
$$

Using $g_{\pi N N}=13.0 \pm 0.1$ [23], corresponding to $q^{2}=m_{\pi}^{2}$, we find

$$
b_{19}=-0.7 \pm 0.4 \text {. }
$$

We can now evaluate Eq. (51) to obtain

$$
G_{P}\left(q_{*}^{2}\right)=8.21 \pm 0.09
$$

which is in good agreement with the best value from non-radiative muon capture [24],

$$
G_{P}\left(q_{*}^{2}\right)=8.7 \pm 1.9
$$

In summary, we have computed the form factors of muon capture by a proton within the framework of the recently derived Ecker-Mojžiš $\mathcal{O}\left(p^{3}\right)$ heavy baryon chiral Lagrangian, and extracted numerical values for some of the Lagrangian's parameters from experimental data. The wave function renormalization factor for nucleons appropriate to this Lagrangian and approach was derived.

\section{ACKNOWLEDGMENTS}

The authors would like to thank Thomas Hemmert for useful conversations. S.S. would like to thank Norbert Kaiser for a useful discussion on renormalization in HBCHPT. This work was supported in part by the Deutsche Forschungsgemeinschaft, the Natural Sciences and Engineering Research Council of Canada, and the NATO International Scientific Exchange Program. 


\section{REFERENCES}

[1] J. Gasser and H. Leutwyler, Ann. Phys. 158, 142 (1984); Nucl. Phys. B 250, 465 (1985).

[2] E. Jenkins and A. V. Manohar, Phys. Lett. B 255, 558 (1991).

[3] G. Ecker, Prog. Part. Nucl. Phys. 35, 1 (1995).

[4] V. Bernard, N. Kaiser and U.-G. Meißner, Int. J. Mod. Phys. E 4, 193 (1995).

[5] A. Pich, Reports on Progress in Physics 58, 563 (1995), hep-ph/9502366.

[6] J. Bijnens, G. Ecker and J. Gasser, Chiral Perturbation Theory in The DA $\Phi$ NE Physics Handbook (2nd. ed.) eds. L Maiani, G. Pancheri and N. Paver (Frascati, 1994).

[7] G. Ecker and M. Mojžiš, Phys. Lett. B 365, 312 (1996).

[8] V. Bernard, N. Kaiser, J. Kambor, and U.-G. Meißner, Nucl. Phys. B 388, 315 (1992).

[9] G. Jonkmans et. al., Phys Rev. Lett. 77, 4512 (1996), nucl-ex/9608005.

[10] H. W. Fearing, R. Lewis, N. Mobed and S. Scherer, in preparation.

[11] Particle Data Group, Phys. Rev. D 54, 1 (1996).

[12] H. W. Fearing, Phys. Rev. C 21, 1951 (1980); D. S. Beder and H. W. Fearing, Phys. Rev. D 35, 2130 (1987).

[13] J. Gasser, M. E. Sainio and A. Švarc, Nucl. Phys. B 307, 779 (1988).

[14] M. Luke and A. V. Manohar, Phys. Lett. B 286, 348 (1992); Yu-Qi Chen, Phys. Lett. B 318, 524 (1993); M. Finkemeier, H. Georgi, and M. McIrvin, hep-ph/9701243.

[15] T.-P. Cheng and L.-F. Li, Gauge Theory of Elementary Particle Physics, Oxford University Press, 1984.

[16] T.-S. Park, D.-P. Min and M. Rho, Phys. Rep. 233, 341 (1993).

[17] G. Ecker, Phys. Lett. B 336, 508 (1994).

[18] Bernard, N. Kaiser, and U.-G. Meißner, hep-ph/9611253.

[19] P. E. Bosted, Phys. Rev. C 51, 409 (1995), and references therein.

[20] A. Delguerra et. al., Nucl. Phys. B 107, 65 (1976); A. S. Esaulov, A. M. Pilipenko and Yu. I. Titov, Nucl. Phys. B 136, 511 (1978); S. Choi et. al., Phys. Rev. Lett. 71, 3927 (1993).

[21] L. A. Ahrens et. al., Phys. Lett. B 202, 284 (1988).

[22] V. Bernard, N. Kaiser, and U.-G. Meißner, Phys. Rev. Lett. 69, 1877 (1992).

[23] V. Stoks, R. Timmermans and J. J. de Swart, Phys. Rev. C 47, 512 (1993).

[24] G. Bardin et. al., Phys. Lett. B 104, 320 (1981). 